\title{
Measurements of the quality of nematic liquid crystal alignment
}

\author{
Iga Ostromęcka, Michał Kwaśny, Paweł Jung, Bartłomiej W. Klus, Urszula A. Laudyn \\ Faculty of Physics, Warsaw University of Technology, Koszykowa 75, 00-662 Warszawa,
}

Received March 17, 2016; accepted March 25, 2016; published March 31, 2016

\begin{abstract}
In this paper we have investigated the alignment of nematic liquid crystal (NLC) molecules in homogenously oriented liquid crystal cells used for nematicons generation. We propose three different methods based on contrast evaluation, linear diffraction and nematicons generation. We showed that LC cell contrast evaluation is not enough from the perspective of nematicons generation. In order to select the best LC cell, all the proposed methods should be combined.
\end{abstract}

The huge availability of liquid crystals mixtures with a variety of parameters (including optical birefringence, sign of electrical and optical anisotropy, thermal stability, etc.) allows easy customization of a designed device for specific conditions and allows for operation in a variable range. Additionally, the use of nonlinear beam propagation of the light allows for the construction of an all-optical waveguide switch [1-9]. A specially designed liquid crystal cell with a properly chosen quality of the alignment layer provides switching and routing of an optical signal without converting it into an electrical signal, possibly the simplest one in operation, running stably and allowing for easy implementation in the existing fiber optic networks. Nowadays, however, a highly important technological problem faced by many researches is the quality of orientation (i.e. alignment layers) used for changing the refractive index distribution within a liquid crystal cell. The orientation of liquid crystal molecules results from the anchoring conditions at the internal surfaces of an LC cell and long-range interaction between molecules [10-12].

Proposed by O. Yaroschuck [13], the method for testing the homogeneity of orientation of liquid crystal molecules is based on analysis of light transmission in crossed polarizers [11]. The method describes the NLC alignment quantitatively but does not give its full assessment. In this work new methods based on linear and nonlinear optical beam propagation through an NLC layer were proposed to investigate the quality of the alignment layer and refractive index distribution.

For experimental verification of the proposed methods, seven independent planar NLC cells of thickness $d=42 \mu \mathrm{m}$ were prepared. The alignment layers ensure planar orientation with initial molecular anchoring at $\theta_{0}=45^{\circ}$ with respect to the $z$-axis (Fig. 1a) Indium tin oxide thin transparent electrodes deposited on the inner sides of cells provide an adjustable low-frequency electric field for out of $y z$-plane steering of molecules. All cells were filled with a 6CHBT NLC.

To describe the quality of NLC alignment, an experimental setup, schematically presented in Fig. 1a, was used.

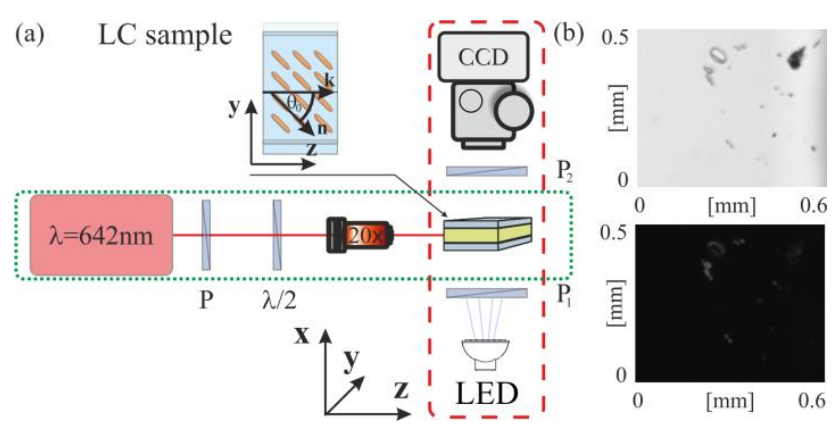

Fig. 1. (a) Geometry of experimental setup; (b) light transmittance through LC cell placed between parallel (top) and crossed (bottom) polarizers.

In the first part of the experiment (marked in the vertical rectangle), the method based on observation of changes in light intensity between crossed polarizers [13] was applied. In contrast to the proposed method, a white light source has been used (broadband LED). Light transmittance through a sample placed between adjustable polarizers mounted above and below the sample, has been measured, respectively. The use of a high resolution CCD camera connected with a microscope lens allowed to record the obtained images of the sample. At first, the two polarizers are crossed and the sample position is precisely adjusted to get minimal transparency, i.e. when the optical axis is parallel to the polarizer $P_{1}$. In this position a digital image is recorded. Then the polarizer $P_{2}$ is rotated by $90^{\circ}$ and the second picture is taken again. Then the ratio $C=\left(I_{\max }-I_{\min }\right) /\left(I_{\max }+I_{\min }\right)$, referred to sample contrast, was measured, where $I_{\max }\left(I_{\min }\right)$ is a mean value of total pixels intensity for a picture taken in parallel (crossed) polarizers respectively. The exposure conditions are limited to avoid saturation of a CCD sensor when both polarizers were set parallel with respect to each other. Typical examples of recorded pictures are presented in Fig. 1b, corresponding to parallel and perpendicular polarizers respectively. The cell with planar anchoring was investigated. Hence, if the alignment is good, 
maximum transmission should be observed between parallel polarizers and minimum - between crossed polarizers, i.e. for good alignment the ratio $\mathrm{C}$ is equal to 1. Thus, any imperfection in the molecules orientation is reflected in lower contrast (quantitatively) and also clearly visible on photos (qualitatively).

In the second part, light beam propagation through a nematic layer (marked in the horizontal rectangle) has been investigated. The laser beam from a linearly polarized laser operated at $\lambda=642 \mathrm{~nm}$ focused onto a beam waist of several micrometers was implemented into the cell with $\boldsymbol{k}$ vector along $z$. The CCD camera was used to collect the light scattered above the cell during light beam propagation.

A linearly polarized light beam with E-field along the $y$ axis and wave vector $\boldsymbol{k} \| z$ was used. In the linear regime, i.e. for low optical power $(<1 \mathrm{~mW})$ the injected Gaussian beam diffracts. For homogeneous orientation of molecules without any imperfections, the beam diameter increases linearly with $z$, remaining its Gaussian intensity. Any disorder in molecular arrangement affects beam evolution and its transverse profile due to changes in the effective refractive index and light scattering on impurities on the surface of an alignment layer. From beam transverse profiles along the distance $z$, the beam width along propagation distance and fit, experimental data with linear function were calculated. The fitting coefficient R-square gives a quantitative alignment quality parameter. Furthermore, for the known value of a beam waist (measured with a beam profiler), wavelength and refractive index, calculations were made of how the Gaussian beam should diverge in theory. The difference between the slope of a theoretical and a fitted experimental line is treated as another parameter which gives information about the molecular order in the area of a diverging beam. The plot examples presenting the compatibility of experimental data to the theoretical curve are presented in Figs. 2a-b. Figure 2a shows the case of uniform diffraction in a cell with no impurities while Fig. $2 b$ shows the case obtained in a cell with poor alignment (presented in Fig. 1b). The recorded images are presented in Figs. 2c-d respectively. The R-square equal to 0.9989 and $\mathrm{a}-\mathrm{a}_{\mathrm{th}}$ equal to $2 \cdot 10^{-5}$ corresponds to the case when the director is oriented unidirectionally, while Rsquare $=0.9974$ and $\mathrm{a}-\mathrm{a}_{\mathrm{th}}=7.4 \cdot 10^{-3}$ occur in the case of random alignment. For both plots, linear fit was performed for the data of the range between $z=100 \mu \mathrm{m}$ to $z=500 \mu \mathrm{m}$.

To validate the proposed methods, nonlinear light beam propagation has been analyzed. In a nonlinear regime, for high enough power, due to reorientation nonlinearity [23 ] the e-beam is self-trapped resulting in spatial solitons (also called nematicons) [2-3], i.e. a beam with unchanged width along the propagation distance (Fig. 2c).
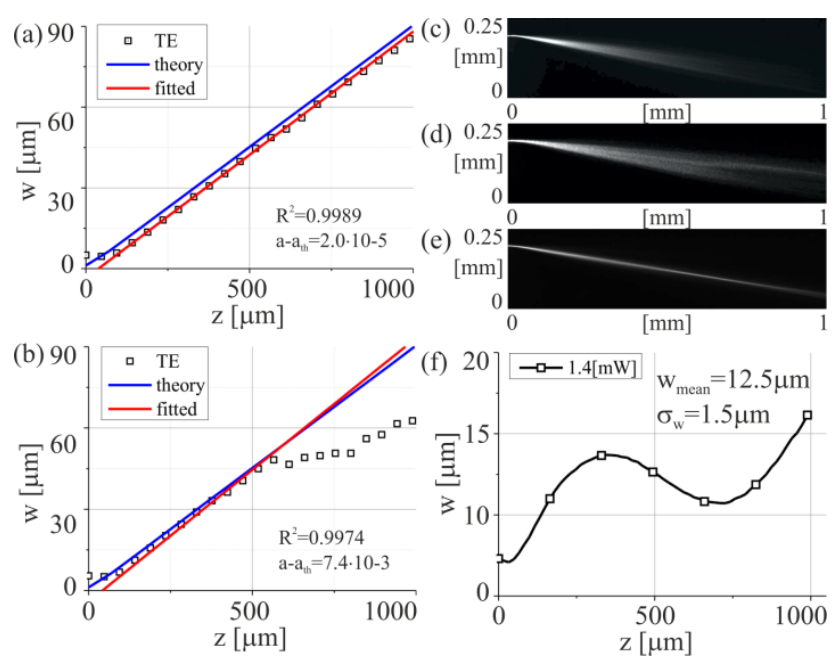

Fig. 2. Linear and nonlinear light beam propagation in an NLC cell: (a) comparison of diffraction angle with theoretical dependences for a cell with no impurities and (b) with some defects; (c-d) corresponding to $(a, b)$ images of the beam evolution along propagation distance $z$ respectively; (e) nematicon propagation for power $\mathrm{P}=1.4 \mathrm{~mW}$; (f) corresponding to (e) plot of beam width vs propagation distance.

The nonlocality of reorientational nonlinearity leads to beam breathing (i.e. the width of a beam oscillates along the propagation distance), with a breathing period dependent on optical power and NLC parameters [14]. An example of beam width evolution for $P=1.4 \mathrm{~mW}$ is plotted in Fig. 2f, corresponding to the photos from Fig. 2e. In this set of experiments, cells with the same thickness (i.e. the same degree of nonlocality [14]) were compared. The samples were filled with the same NLC material which determines that the obtained nematicons should have similar parameters (i.e. should have the same width along $z)$. Therefore the mean width value and its standard deviation, described as $\sigma$ were used to compare the cells.

Figure 3 presents the results of contrast and linear diffraction measurements. As visible in Fig. 3a, only one cell (no. 5) has a considerably lower contrast, while all other cells can be marked as "very good". On the contrary, looking at the R-square parameter (Fig. 3b) only cell no. 2 can be marked as "imperfect". However, the differences in parameter R-square are not very significant. The greatest diversity is reflected in the $\mathrm{a}-\mathrm{a}_{\mathrm{th}}$ parameter. Combining the results $\left(\mathrm{R}^{2}\right.$ and $\left.\mathrm{a}-\mathrm{a}_{\mathrm{th}}\right)$, it is visible that the best quality is assigned to cell no. 3. All other cells despite having a high contrast (except no. 5) show some imperfections in the analysis of linear diffraction. 


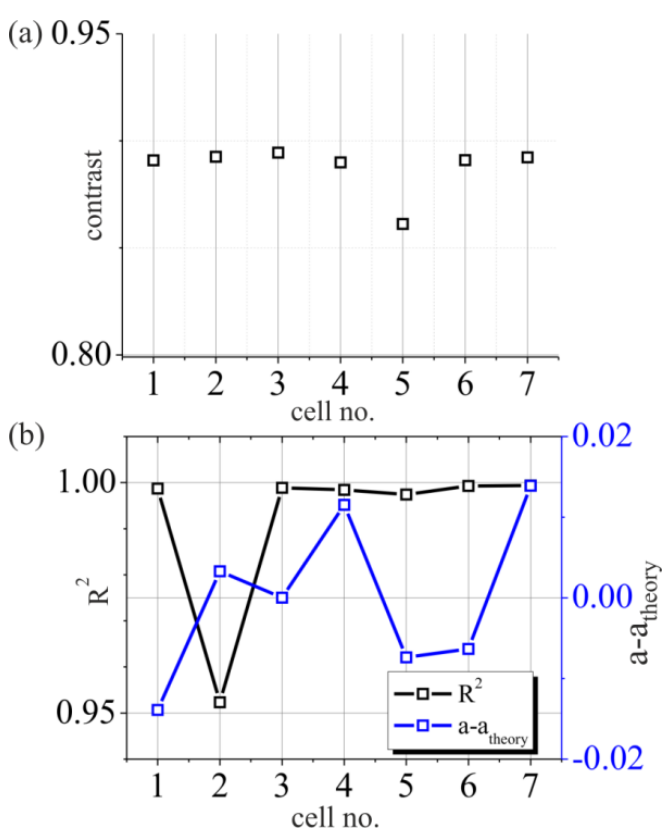

Fig. 3. Comparison of liquid crystal cells: (a) contrast ratio; (b) linear beam propagation $\mathrm{R}^{2}$ parameter and difference between observed experimentally diffraction angle and theoretical dependence.

The results obtained from both graphs (Fig. 3a-b) lead to a conclusion that cell no. 3 is characterized with the best alignment quality, cells no. 1, 5 and 7 have the worst aligning parameters, while all others are of good quality.

The verification of this has been made by increasing the beam power and analysing the nematicon propagation The mean width for different optical powers and its standard deviation are plotted in Fig. 4a and Fig. 4b respectively. Indeed, in cell no. 3, the light localization for power $P=1.4 \mathrm{~mW}$ was obtained. For this power, in cells no. 1, 5 and 7 the beam width is still considerably higher (Fig. 4a), also the standard deviation reaches the maximum value. Even though cells no. 1 and 7 are characterized by a good contrast, the diffraction patterns show the greatest deviation from theory for a Gaussian beam, simultaneously the beam width calculated for $P=1.4 \mathrm{~mW}$ is larger than in other cells. By taking into consideration all of the calculated indicators, it can be noted that the best parameters were achieved in cells no. 3 and no. 6. Those cells are characterized by very high contrast ratio, also in both cases the $\mathrm{R}^{2}$ parameter value is close to 1 . The diffraction angle is in good agreement with theoretical dependence.

To conclude, three methods are proposed for measuring the alignment quality in planar liquid crystal cells. They are based on a combination of contrast measurements and analysis of linear and nonlinear beam propagation. Our results demonstrate that proposed methods can be used in quality evaluation of LC cells to be used as a waveguide for self-guided beams.
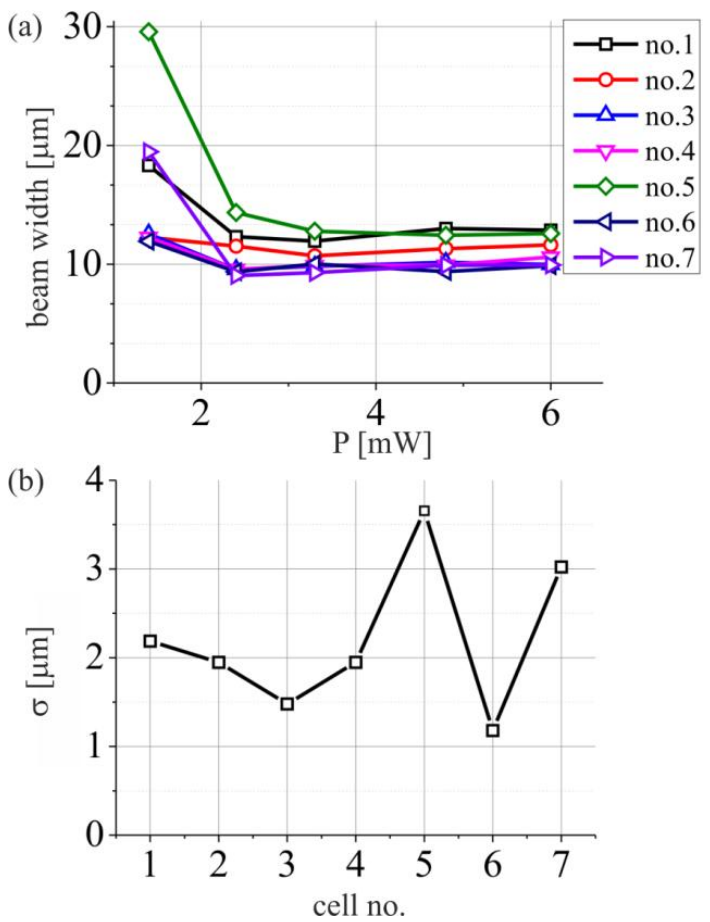

Fig. 4. (a) Beam waist evolution in nonlinear regime; (b) Standard deviation of the beam width for $P=1.4 \mathrm{~mW}$.

This work was financially supported by the National Centre for Research and Development by the grant agreement LIDER/018/309/L-5/13/NCBR/2014.

\section{References}

[1] S. Trillo, W. Torruellas, Spatial Solitons (Springer, Berlin 2001).

[2] G. Assanto, Nematicons (Wiley, 2012).

[3] M. Peccianti, G. Assanto, Phys. Rep. 516, 147 (2012).

[4] Ya.V. Izdebskaya, J. Rebling, A.S. Desyatnikov, G. Assanto, Yu.S. Kivshar, Opt. Express 20, 24701 (2012).

[5] A. Piccardi, et al., Appl. Phys B 104, 5 (2011); doi: 10.1007/s00340011-4675-0.

[6] S.V. Serak, N.V. Tabiryan, M. Peccianti, G. Assanto, IEEE Phot. Tech. Letters 18(12), 1287 (2006).

[7] A. Piccardi, U. Bortolozzo, S. Residori, and G. Assanto, Opt. Lett. 34, 6 (2009); doi: 10.1364/OL.34.000737.

[8] U.A. Laudyn, M. Kwasny, M.A. Karpierz, Opt. Comm. 283, 1463 (2010).

[9] U.A. Laudyn, M.A. Karpierz, Appl. Phys. Lett. 103, 221104 (2013).

[10] P. Yeh, C. Gu, Optics of Liquid Crystal Displays (Wiley, 1999).

[11] I.C. Khoo, Liquid Crystals (Wiley, 2007).

[12] Q. Li, Nanoscience with Liquid Crystals (Springer, 2014).

[13] L. Vretik, O. Yaroshchuk, V. Zagnii, V. Kyrychenko, V. Syromyatnikov, Liquid crystalline organic compounds and polymers as materials of XXI century: From synthesis to applications, A. Iwan and E. Schab-Balcerzak (Ed.), Transworld Research Network, Chapt. 6, pp.153-189 (2011)

[14] C. Conti, M. Peccianti, G. Assanto, Phys. Rev. Lett. 92, 113902 (2004). 